\title{
Necesidades de formación profesional en el ámbito de la primera infancia: Percepción y aportes del estudiantado' ${ }^{1}$
}

\author{
Needs of Professional Formation in the field of Early Childhood: Perception and \\ Contributions from the Students ${ }^{2}$
}

\author{
Rocío Castillo-Cedeño 3 \\ Universidad Nacional \\ Heredia, Costa Rica \\ rociocastillocedeno@gmail.com
Patricia Ramírez-Abrahams ${ }^{4}$
Universidad Nacional
Heredia, Costa Rica \\ pramirezabrahams@gmail.com \\ Lilian Susana Ruíz-Guevara \\ Universidad Nacional \\ Heredia, Costa Rica \\ susanaruizguevara@gmail.com
}

Recibido 19 de octubre de 2015 • Corregido 16 de octubre de 2016 Aceptado 24 de noviembre de 2016

\begin{abstract}
${ }^{1}$ Los datos y el análisis que se presentan en este artículo son parte de una experiencia de investigación desarrollada en el proyecto de investigación Primera infancia: Hacia una propuesta de formación profesional contextualizada y pertinente (Ramírez, Ruiz y Castillo, 2015), de la División de Educación Básica del Centro de Investigación y Docencia en Educación de la Universidad Nacional.

${ }^{2}$ The analysis and data presented in this article are part of a research experience carried out in the investigation project titled Early Childhood: Toward a Proposal of a Professional, Contextualized, and Pertinent Formation (Ramírez, Ruiz y Castillo, 2015), run by the Basic Education Division at the Center of Research and Teaching in Education of the National University of Costa Rica.

${ }^{3}$ Doctora en Educación con especialidad en Mediación Pedagógica. Magíster en Administración Educativa y en Psicopedagogía. Profesora y bachiller en Ciencias de la Educación con énfasis en Educación Preescolar, Universidad Nacional. Ha participado activamente en procesos de formación de personal formador, principalmente, en el diseño e implementación de cursos de pedagogía a distancia, en la Universidad Estatal a Distancia. Durante cinco años se desempeñó como decana de la Facultad de Educación de la Universidad De La Salle Costa Rica y trabajó durante un año como Vicerrectora Académica en esta misma universidad. Actualmente, se desempeña como docente e investigadora en el Centro de Investigación y Docencia en Educación de la Universidad Nacional de Costa Rica. Gestora de la carrera de Pedagogía con énfasis en Educación Preescolar. Autora del libro: La conciencia cósmica en la primera infancia. Reflexiones paradigmáticas, propuestas biopedagógicas y un acercamiento a la espiritualidad de las niñas y los niños.

${ }^{4}$ Máster en Pedagogía con énfasis en Atención y Desarrollo de la Primera Infancia en la Universidad Nacional. Licenciada en Pedagogía con énfasis en Educación Preescolar, en la Universidad Nacional. Trabajó como responsable académica del énfasis en Primera Infancia, en la Maestría en Pedagogía de la División de Educación Básica en el periodo 2010-2012. Ha sido directora de trabajos finales de graduación durante 14 años. Actualmente se desempeña como académica en la División de Educación Básica, integra la Comisión de Trabajos Finales de Graduación de la División y trabaja en el proyecto de investigación "Primera Infancia: Hacia un perfil de formación contextualizado y pertinente" hace 2 años. Durante 4 años ha sido miembro del Comité de Gestión Académica de la Maestría en Pedagogía con énfasis en Desarrollo y Atención Integral a la Primera Infancia.

${ }^{5}$ Doctora en Educación, Máster en Administración y Licenciada en Educación preescolar, laboró por más de 14 años como docente de preescolar con niños y niñas desde los 2 hasta los 6 años, ha laborado para la DEB, UNA por 15 años como académica e investigadora. Ha pertenecido al equipo que lidera los procesos de acreditación de las carreras de educación de la DEB, desde el año 2000, actualmente coordina estos procesos para las 3 carreras. Ha participado en proyectos de investigación en temáticas relacionadas con perfil docente, transición de preescolar a primaria y primaria a secundaria, evaluación de los aprendizajes en el ámbito universitario, entre otros. Tiene publicaciones en diferentes revistas en temas relacionados con primera infancia, autoevaluación, evaluación de aprendizajes, transición, investigación en la formación docente, entre otros.
\end{abstract}


doi: http://dx.doi.org/10.15359/ree.21-1.9

URL: http://www.una.ac.cr/educare

CORREO: educare@una.cr

Resumen: La información recopilada tiene el propósito de identificar las necesidades de formación profesional en el ámbito de la primera infancia, según la percepción del estudiantado que cursa el V y VI nivel de la Licenciatura en la Carrera de Pedagogía con énfasis en Educación Preescolar. El objetivo de este estudio fue conocer sus percepciones sobre las necesidades de formación docente que se presentan en cuanto a la atención y educación en la primera infancia, para lo cual se considera su experiencia laboral, las experiencias y cursos que brinda la carrera en relación con dicho grupo etáreo, las necesidades de formación y los aspectos que la carrera debe mejorar para brindar una formación docente acorde con las necesidades y demandas actuales en el campo de la atención y educación para la primera infancia. Esta investigación tuvo un diseño metodológico exploratorio. Participan en la indagación, los grupos del nivel de Licenciatura de la carrera mencionada. El instrumento que se utiliza para la recopilación de la información es un cuestionario con preguntas abiertas relacionadas con las necesidades de formación y las experiencias concretas vivenciadas en este proceso. El análisis de datos de este estudio es descriptivo. Después se complementa la información recopilada con la investigación teórica, los aportes del proceso de revisión del Plan de estudios de la carrera de preescolar y los conocimientos construidos en la experiencia del proyecto de investigación Primera infancia: Hacia una propuesta de formación profesional contextualizada y pertinente. Entre las principales conclusiones: la mayoría de las estudiantes expresan que su formación carece de instrumentación profesional para el trabajo con la niñez desde el nacimiento hasta los cuatro años y la importancia de trabajar los procesos de intervención pedagógica en otras áreas que prioricen la inclusión del ciclo materno infantil y temáticas de estimulación temprana. Esta sistematización y análisis de la percepción del estudiantado ofrecen un insumo a considerar para el Plan de Estudios de la Carrera de Educación Preescolar, dado que estos resultados podrían colaborar en su revisión a la luz de las percepciones y vivencias del estudiantado, aportes fundamentales para construir una propuesta de formación docente contextualizada y pertinente.

Palabras claves: Necesidades: formación docente; primera infancia; percepción; vivencias; contextualización y pertinencia.

\begin{abstract}
The analysis and information presented in this article are part of an experience developed in the research project Primera infancia: hacia una propuesta de formación contextualizada y pertinente [Early Childhood: Toward a Proposal of a Professional, Contextualized, and Pertinent Formation] of the Basic Education Division at the National University of Costa Rica. The information was collected with the purpose of identifying the needs for professional formation on the field of early childhood, through the perception and contributions of students in levels $V$ and $\mathrm{VII}$ of their undergraduate degree in the Pedagogy career with a concentration in preschool education. This is an exploratory-type investigation aiming to know the opinions and perceptions of the students in relation with the process they go through at the university. For it, we used a questionnaire of seven open questions related to the needs of formation and concrete experiences lived during this process. As a result from this study, the analysis had a descriptive conclusion when exploring the main needs of the students. The information collected was complemented with the theory of investigation known, the contributions for the review of the whole preschool study plan, and all the knowledge built during the research project aforementioned. One of the main findings is that most of the students expressed that their formation lacks of the professional instrumentation to work with children aged zero to four years. They also said that it is very important to start working in a similar process on the earlier stages Pedagogy covers like maternal cycle and early stimulation. This systematization and analysis of the students' perception is an effort to support the review of the study plan for the Pedagogy career with concentration in preschool education. The help of the students' perceptions and experiences is a fundamental contribution in order to build a contextualized and relevant proposal; this is the main part of the research project aforementioned.
\end{abstract}

Keywords: Needs, professional formation, early childhood, perception, contextualization, relevance and experiences. 


\section{Ideas iniciales}

La temática de las necesidades de formación profesional en el ámbito de la primera infancia desde las percepciones del estudiantado es de gran relevancia para la revisión y análisis de la propuesta de formación que brinda la Universidad Nacional de Costa Rica en materia de pedagogía con énfasis en educación preescolar. Tarea fundamental, al tratarse de una carrera acreditada, que requiere que su propuesta formativa se revise constantemente, considerando las tendencias actuales en elámbito de estudio, las necesidades sociales, económicas, culturales y pedagógicas que orientan esta formación. Para ello, es urgente explorar las diferentes percepciones de los principales agentes que participan en el proceso de formación. Por lo tanto, la opinión del profesorado y el estudiantado es de gran importancia, debido a que son quienes tienen información derivada de vivencias concretas, fundamentales como marco de análisis de las propuestas vigentes.

En este caso, se ha priorizado en la percepción del estudiantado por considerarlo el actor principal del proceso educativo.

Estudios realizados (Peralta, 2005; Zapata y Ceballos, 2010) apuntan a la importancia de la atención y educación de la niñez en la primera infancia, pues esta etapa constituye un momento crucial en la vida humana, en la que se dan cambios que afectan significativamente el desarrollo de las personas.

Hay tres puntos fundamentales que pueden justificar la inversión estatal y el interés político en la educación en la primera infancia:

1. El avance en el campo de las neurociencias que ha señalado que en el primer año de vida suceden la mayoría de cambios a nivel cerebral, incluso los estudios afirman que en esa edad se ha formado hasta un $85 \%$ del cerebro humano.

2. La necesidad de que los niños y las niñas tengan experiencias cognitivas y afectivas que afecten significativamente su desarrollo para garantizar un desarrollo adulto adecuado.

3. La importancia de promover espacios educativos de alta calidad en estos primeros años de vida, con el fin de asegurar el desarrollo integral de todas las personas independientemente de sus condiciones sociales (culturales, comunales e históricas) e individuales (físicas, afectivas, cognitivas, lingüísticas y motoras).

Por estas razones y otras que señalan las investigaciones (Elvir y Asensio, 2006; Eming y Fujimoto 2004; Myers, 1999; Sauma, 2012), la formación de las personas docentes encargadas de atender a la niñez en esta etapa de vida es un tema de discusión académica, en relación con la conformación de una propuesta de formación que atienda las demandas y necesidades de un contexto social, histórico y político, que requiere de un escenario educativo que considere a la primera infancia como parte esencial del desarrollo humano. 
doi: http://dx.doi.org/10.15359/ree.21-1.9

URL: http://www.una.ac.cr/educare

CORREO: educare@una.cr

Uno de los factores que apuntan investigaciones como la de Peralta (2005) es que la formación del profesorado se ha centrado en edades de 4 a 6 años, dentro de la cobertura de programas de carácter formal. Las iniciativas de cuidado para la población menor de 4 años están bajo la responsabilidad de otro tipo de servicios gubernamentales que no han logrado la cobertura necesaria para atender a toda la población infantil que lo requiere. Esto se evidencia en datos del Programa Estado de la Nación (2013) cuando señalan que la oferta para "Ios niños de 4 años es baja: 38,1\% según el Censo 2011. En los centros públicos la asistencia es alta entre los menores de 1 año (70,9\%) y los mayores de 4 años (más del 70\%), no así entre los de 1 a 3 años (menos del 50\%)" (p. 77).

El Programa Estado de la Nación (2013) señala datos importantes que justifican un cambio en la formación del profesorado del área de primera infancia. Esta realidad se expresa desde políticas esenciales como la Constitución Política Costarricense, en la que se afirma que el Estado está obligado proveer oferta de programas y servicios para la niñez a partir de los dos meses de edad; sin embargo, a la fecha, ha logrado una cobertura aproximada de un 70,9\% para la población de 4 años, que asiste, en su mayoría, al servicio público de educación preescolar en el nivel interactivo II (servicio educativo formal para niños de 4 años y 3 meses, hasta los 5 años y 3 meses).

En el caso de los niños y las niñas menores de 4 años, los datos invitan a una reflexión en relación con la atención y educación que se brinda en el país. Por ejemplo, en zonas de mayor desarrollo de las provincias de la gran área metropolitana, los servicios y programas para menores de 4 años están ubicados alrededor de un $50 \%$ en oferta privada, en guarderías y centros maternales (Programa Estado de la Nación, 2013). En la información recopilada con las estudiantes universitarias que cursan la carrera en Pedagogía con énfasis en Educación Preescolar de la División de Educación Básica (DEB), se encuentra que, en su mayoría, cuando encuentran su primer trabajo es con niños menores de 4 años y en instituciones como las mencionadas anteriormente: de carácter privado. Por tal razón, las necesidades de formación, según lo expresado por el estudiantado, refieren a la importancia de retomar esta área en los planes de estudio, como un punto crucial para la preparación del personal docente.

\section{Reflexiones teóricas en relación con la formación universitaria del personal docente de educación preescolar}

La reflexión en torno al tema de la formación docente implica, necesariamente, el estudio de las diferentes variables que intervienen en la construcción de un perfil adecuado para la realidad sociocultural en la cual se gesta la propuesta. A nivel global existe una serie de principios y tendencias que orientan la formación. Villegas-Reimers (2002) señala que "según la nueva concepción del desarrollo profesional del docente, el trabajo del maestro se ha reconocido como algo bastante más complejo que simplemente transmitir información específica y saber enseñar, razón por la cual se ha adoptado una perspectiva mucho más amplia e inclusiva" (p. 72). 
Esta concepción compleja se ubica en un paradigma holístico, la cual visualiza una formación integral que considere la triada mente, cuerpo y espíritu; esto. por lo tanto, apunta a la importancia de priorizar múltiples saberes tanto conceptuales, procedimentales como actitudinales.

Desde este marco de referencia, para Villegas-Reimers (2002) se requiere una propuesta de formación docente que potencie el conocimiento:

- Pedagógico general: implica el conocimiento de los entornos de aprendizaje y todas las dimensiones que lo componen.

- Contenido pedagógico específico: que contesta la pregunta de cómo enseñar una disciplina, la comprensión del estudiantado y conocimiento del currículo

- El contexto del estudiantado: la comunidad, familia y las instituciones educativas.

- Cómo salvar la brecha entre la teoría y la práctica

- Evaluación externa del aprendizaje

- Estrategias, técnicas y herramientas diseñadas para crear y sustentar una comunidad o entorno del conocimiento

- Destrezas y predisposición para trabajar atendiendo la diversidad

- Actitudes que apoyan la justicia política y social

- Saber cómo aplicar la tecnología al currículo.

Con respecto a las tendencias específicas en relación con la formación de docentes para la atención y educación en la primera infancia se plantean transformaciones importantes debidas a las demandas de una sociedad contemporánea, cuyo estilo de vida ha inducido cambios en la estructura familiar; la organización económica, política e histórica; la posición de la mujer en la sociedad, y la concepción del niño y la niña como sujetos de derechos (Peralta 2005; Sauma, 2012; Zapata y Ceballos, 2010); también, las transformaciones epistemológicas referidas a cómo se enseña y cómo se aprende.

En relación con lo anterior, en el ámbito de primera infancia, ello ha transcurrido por variedad de enfoques. El enfoque asistencial, que prioriza la satisfacción de necesidades básicas (alimentación, salud, cuido) de la niñez menor de 6 años como fundamentales para el desarrollo $y$, por ende, para que el niño y la niña aprendan mejor; el de preescolaridad, que enfoca las acciones pedagógicas a la preparación de los niños y las niñas para el futuro escolar; y el de atención, educación y cuido en la primera infancia, que apunta a que independientemente de las condiciones en que se desarrolle la persona, tiene derecho a una educación oportuna y pertinente desde su nacimiento (Peralta, 2005). 
doi: http://dx.doi.org/10.15359/ree.21-1.9

URL: http://www.una.ac.cr/educare

CORREO: educare@una.cr

En la docencia para la primera infancia incide significativamente el avance del conocimiento acerca del desarrollo humano, los recientes descubrimientos en relación con el funcionamiento cerebral, el pensamiento complejo y todo lo que sucede en la niñez temprana como parte esencial de un desenvolvimiento de la persona durante toda su vida de forma saludable y feliz. Esto ha implicado la incorporación de la pedagogía en un campo que estaba dominado por el asistencialismo y el cuidado como la forma socialmente aceptada de atención a la primera infancia.

De ahí, la importancia de la resignificación de la niñez en la formación docente, incluyendo los retos que plantea una pedagogía centrada en el desarrollo pleno y armónico de la persona en relación sistémica con el medio que le rodea y las interacciones afectivas y cognitivas que le influyen y afectan. Todo lo anterior, en el reconocimiento de los componentes de su desarrollo integral. Entonces, en este contexto:

El momento actual es de desafío formativo y resignifica la actuación, en relación a procurar la pertinencia del conocimiento y el conocimiento en procura de transformaciones de la realidad, es decir, en transformaciones del pensar, del conocer y del sentir por ello implica de un Pensamiento Complejo. (Amador, 2007, p. 47)

Esta disyuntiva de la pedagogía y su papel en la educación de la niñez desde el nacimiento provocan, necesariamente, un cambio en la visión de los programas de formación docente, que en el ámbito universitario público ha predominado en las últimas décadas. Se exige que haya un proceso de formación mediado por una profunda capacidad de reflexión sobre las propias prácticas docentes y experiencias andragógicas en el aula universitaria, para la solución pedagógica de las situaciones que emergen en la cotidianeidad de un ambiente educativo en que los grupos profesionales en formación se enfrentarán a un estudiantado cuyas edades empiezan desde el nacimiento hasta los 4 años. Chaves, Díaz, García, Rojas, Solís (2007) sugieren entonces "espacios académicos donde adquieran formación metodológica y teórica dirigida al diálogo permanente con equipos de investigación" (p. 26).

Para ello, se espera una formación docente que conduzca a una educación en virtud de lograr:

Una persona profesional de la pedagogía y de la investigación que asume el rol de sujeto mediador entre el objeto de conocimiento y el sujeto que aprende y reconstruye; como un orientador u orientadora y guía del aprendizaje, que asesora, crea, facilita y propone situaciones problemáticas. (Zapata y Ceballos, 2010, p. 1071)

Este requerimiento es necesario en un contexto, como se ha afirmado antes, donde no existe la cobertura ni la oferta pública suficiente para la atención integral y educación de la 
niñez temprana menor de 4 años (Ruiz, Castillo, Ramírez y Urdaneta, 2015). Esta necesidad podría solventarse a partir de un proceso dialógico entre el conocimiento práctico y el teórico que se encuentra en los ambientes de cuidado, educación y atención de la niñez, traducido en prácticas contextualizadas y pertinentes, con el fin de solidificar, con herramientas elaboradas y recuperadas de procesos de investigación, la formación y desarrollo profesional de docentes que trabajen con esta población.

Esto implica, desde la formación de personal formador, el autoconocimiento de las necesidades propias de formación con la conciencia de que su propio quehacer debe ser pensado y reflexionado. Esto último, con la firme convicción de que su práctica docente puede mejorar. Esta autoformación y desarrollo profesional permite la incorporación de nuevos conocimientos y mejores intervenciones en la práctica cotidiana del estudiantado universitario que se inserta en el campo de trabajo con niños y niñas menores de 4 años, por lo tanto, es necesario que la carrera universitaria le haya provisto de los conocimientos e instrumentación para el abordaje pedagógico de esta población.

En relación con lo anterior, interesa destacar que la educación inicial del nuevo siglo, los avances en las neurociencias y en el cómo se aprende en la niñez han abierto un panorama nuevo en cuanto a la concepción de niño y niña que se tenía tradicionalmente. Un claro ejemplo son las investigaciones que afirman que el desarrollo de la actividad cerebral inicia desde el útero y que los primeros dos años de vida son fundamentales en el crecimiento neuronal. Otro descubrimiento importante que se ha hecho es que los niños y las niñas en condiciones de pobreza extrema podrían afectar su desarrollo si no se les atiende antes de los 18 meses de vida (Peralta, 2005).

Estos datos ponen de manifiesto la necesidad de intervenir desde edades tempranas en los procesos de educación no formal y formal de la niñez. Esta resignificación de la importancia del aprendizaje en la primera infancia es la que lleva a plantear la diferencia entre los viejos y los nuevos paradigmas, cómo se visualiza la educación de la infancia y, en consecuencia, asumir las repercusiones que tienen en la formación docente.

Se parte de una dimensión interactiva del aprendizaje, en la que se reconoce que los niños y las niñas se desarrollan en múltiples contextos que les proveen de material para aprender. Este proceso no es solo a un nivel cognitivo individual, sino también social, en el que la influencia de los otros proporciona herramientas para la construcción significativa de los conocimientos. Por tanto, es necesaria la reflexión a la luz de la formación docente y cómo se abordan las necesidades y requerimientos de las poblaciones en la primera infancia.

Estas reflexiones son las que motivan el presente estudio. 
doi: http://dx.doi.org/10.15359/ree.21-1.9

URL: http://www.una.ac.cr/educare

CORREO: educare@una.cr

\section{Metodología}

Esta investigación se desarrolla dentro de la etapa de diagnóstico del proyecto Primera Infancia: Hacia una propuesta de formación contextualizada y pertinente (Ramírez, Ruiz y Castillo, 2015). En esta se buscó indagar las necesidades de formación desde el estudiantado, que en ese momento cursaba los últimos niveles de la carrera, así como las necesidades que expresan los profesionales y las profesionales en servicio. En este artículo se hace referencia a las percepciones expresadas por las estudiantes consultadas de la carrera de Pedagogía con énfasis en Educación Preescolar, de la División de Educación Básica (DEB) de la Universidad Nacional.

\section{Tipo de estudio}

Este estudio se desarrolló con el propósito de identificar las percepciones del estudiantado del nivel V y VI de la carrera de Pedagogía con énfasis en la Educación Preescolar. La investigación es de tipo exploratorio, porque se pretendió diagnosticar las necesidades de formación que expresan un grupo de estudiantes en el nivel de licenciatura. El estudio induce a las participantes a reflexionar acerca de cuáles consideran son las áreas y conocimientos que a lo largo de su formación universitaria no han sido abarcados en la implementación del plan de estudios de la carrera.

\section{Participantes}

La investigación se realizó con 43 estudiantes (en su totalidad mujeres) de V y VI nivel de la Licenciatura en Pedagogía con énfasis en Educación Preescolar, a finales del año 2013, principios del 2014 y 2015. La población participante es escogida porque ha vivido la mayoría del proceso formativo propuesto en el plan de estudios de la carrera, por ello, pueden aportar información a partir de sus experiencias concretas. Este fue un punto fundamental porque se utiliza el criterio de accesibilidad a las participantes, debido a que en el momento del estudio fueron estudiantes regulares, que estudiaban las asignaturas de la licenciatura. Durante 3 cursos lectivos se implementa el espacio de consulta para conocer su percepción en relación con las necesidades de formación que han presentado. Otro de los criterios que se utiliza es la selección a conveniencia, porque el estudiantado que participa ha cursado todo el plan de estudios de Pedagogía con énfasis en Educación Preescolar, vigente desde el año 2006.

\section{Instrumentos}

Para la recopilación de la información se elabora un cuestionario que cuenta con 7 preguntas abiertas destinadas a explorar: 
- La población y su experiencia laboral en el ámbito de la educación y atención de la primera infancia, principalmente con la niñez menor de 4 años.

- Las experiencias y cursos que brinda la carrera en relación con la niñez menor de 4 años.

- Las necesidades de formación en cuanto a la parte teórica, metodológica. curricular y de trabajo con la familia y comunidad.

- Los aspectos que la carrera debe mejorar para brindar una formación profesional acorde con las necesidades y demandas actuales en el campo de la atención y educación para la primera infancia.

Todos los datos obtenidos a partir de cuestionario, se sistematizan-considerando los principales insumos teóricos investigados y los aportes de la revisión del plan de estudios de la carrera en Pedagogía con énfasis en Educación Preescolar que se imparte en la División de Educación Básica de la Universidad Nacional.

\section{Análisis de datos}

La información recopilada se sistematiza por medio de frecuencias en las respuestas dadas por las participantes. El análisis tiene un alcance descriptivo que se complementa con la revisión de fuentes bibliográficas y los aportes ofrecidos por el proceso de revisión del Plan de Estudios de la Carrera en Pedagogía con énfasis en Educación Preescolar, además, del conocimiento construido en el proceso de investigación del proyecto de Primera Infancia: Hacia una propuesta de formación contextualizada y pertinente (Ramírez et al., 2015) que se desarrolla en la División de Educación Básica de la Universidad Nacional.

\section{Resultados, vivencias y percepciones}

\section{La población consultada: Acercamiento a su experiencia laboral}

Con respecto a la caracterización específica de la población en función de su experiencia laboral, en el ámbito de la primera infancia se puede indicar que un $58 \%$ se encuentra trabajando en preescolar o en un área relacionada con atención y educación de niños pequeños $(0$ a 4 años), lo que nos deja a un porcentaje de $42 \%$ que actualmente no labora (Figura 1). 
doi: http://dx.doi.org/10.15359/ree.21-1.9

URL: http://www.una.ac.cr/educare

CORREO: educare@una.cr

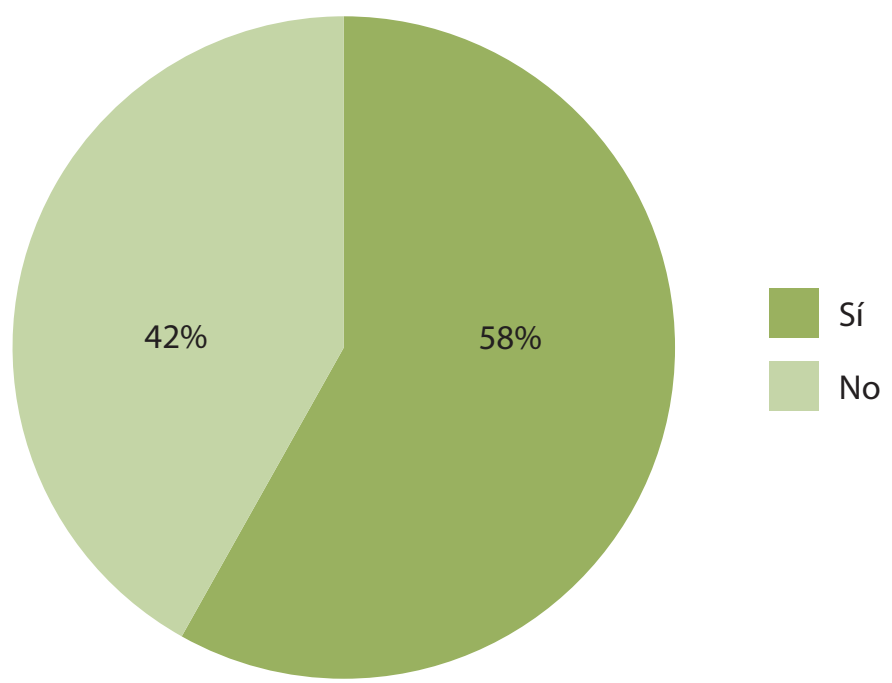

Figura 1: Estudiantes que laboran. Elaboración propia.

Este dato es importante, porque permite observar que las participantes expresan que en su mayoría han trabajado con niños y niñas menores de 4 años. El Plan de estudios en Pedagogía con énfasis en Educación Preescolar (2013) tiene como población meta la niñez desde el nacimiento hasta los 8 años de edad; no obstante, las estudiantes consultadas afirman, como necesidad, un mayor conocimiento sobre el desarrollo humano en los primeros 4 años de vida (Tabla 1).

Esto apunta a la importancia de una formación docente que considere que el contexto de desempeño laboral en el que desenvuelve una persona profesional en primera infancia, que depende mucho también de las políticas laborales de un país y la situación de la mujer trabajadora (Sauma, 2012). En Costa Rica, la población infantil ingresa a servicios de cuido, atención y educación desde los tres meses de edad. Esta situación plantea la pregunta: ¿La estudiante universitaria está preparada para enfrentar una realidad curricular en la que su estudiante es un bebé? (Ramírez et al., 2015). Esto se reafirma con el dato que se presenta en relación con la experiencia laboral de la población consultada (Figura 2).

En lo que respecta a la experiencia laboral con niñez menor de 4 años, las personas cuestionadas responden que un $79 \%$ sí ha trabajado con esta población, con un alto índice de experiencia entre los 2 y 4 años de edad, y un porcentaje de $21 \%$ indica no haber trabajado con menores de 4 años (Figura 2). Los datos anteriores confirman una de las necesidades de formación expresadas en los momentos de revisión del plan, específicamente en las ideas compiladas en la sistematización del proceso diagnóstico de la carrera en los años 2009-2013 y en las observaciones de los sujetos pares como resultado del proceso de reacreditación de 
la carrera (Urdaneta, 2013), en las que se indica la necesidad de incorporar, en la formación, procesos en los que se les brinde a las estudiantes conocimiento teórico-práctico para el trabajo con niñez menor de 3 años.

Esto último, aunado a la realidad que las estudiantes participantes reportan, donde indican que sus primeras experiencias laborales las tienen con la población mencionada. Esto se muestra en los resultados obtenidos en la recopilación de la información.

El rango de edades, expresado por las estudiantes, con la que mayoritariamente se ha trabajado es de 2 años hasta los 4 años, confirmado por el 79\% que afirma tener experiencia laboral con estas edades. Entonces se les pregunta si la carrera les ha ofrecido, en su formación, insumos para este desempeño en el campo profesional.

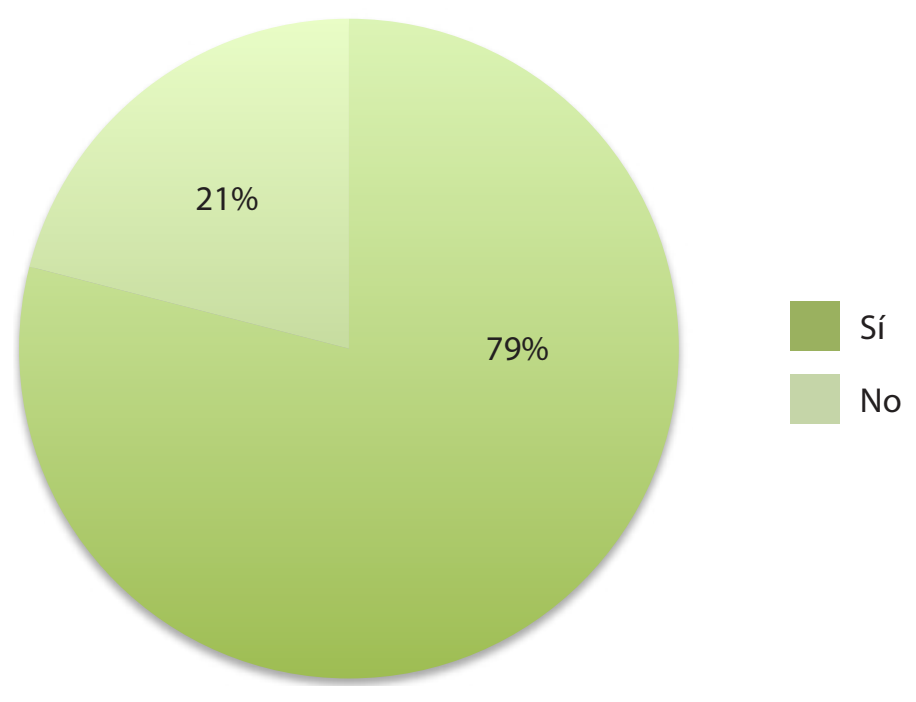

Figura 2: Estudiantes que poseen experiencia laboral con la niñez menor de 4 años. Elaboración propia.

\section{Experiencias de formación brindadas durante la carrera}

Con respecto a las experiencias de formación brindadas por la carrera en relación con la educación y atención de la niñez menor de 4 años, es importante señalar que un $89 \%$ de la población entrevistada considera que en la carrera han tenido experiencias de formación relacionadas con la intervención pedagógica, observación, proyectos u otras asignaciones de los cursos que tuvieran que ver con menores de 4 años, solamente un $8 \%$ considera que no y 
doi: http://dx.doi.org/10.15359/ree.21-1.9

URL: http://www.una.ac.cr/educare

CORREO: educare@una.cr

un porcentaje de 3\%, no responde (Figura 3). Este $89 \%$ es un dato que llama la atención, porque se contrapone en relación con las necesidades de formación expresadas por la población entrevistada cuando afirma que requiere de mayor acercamiento teórico práctico en el trabajo con niñez menor de 4 años. Invita a la reflexión en relación con si el requerimiento hacia el plan de estudios va orientado hacia las experiencias de práctica en las aulas y un mayor énfasis en intervenciones pedagógicas en espacio.

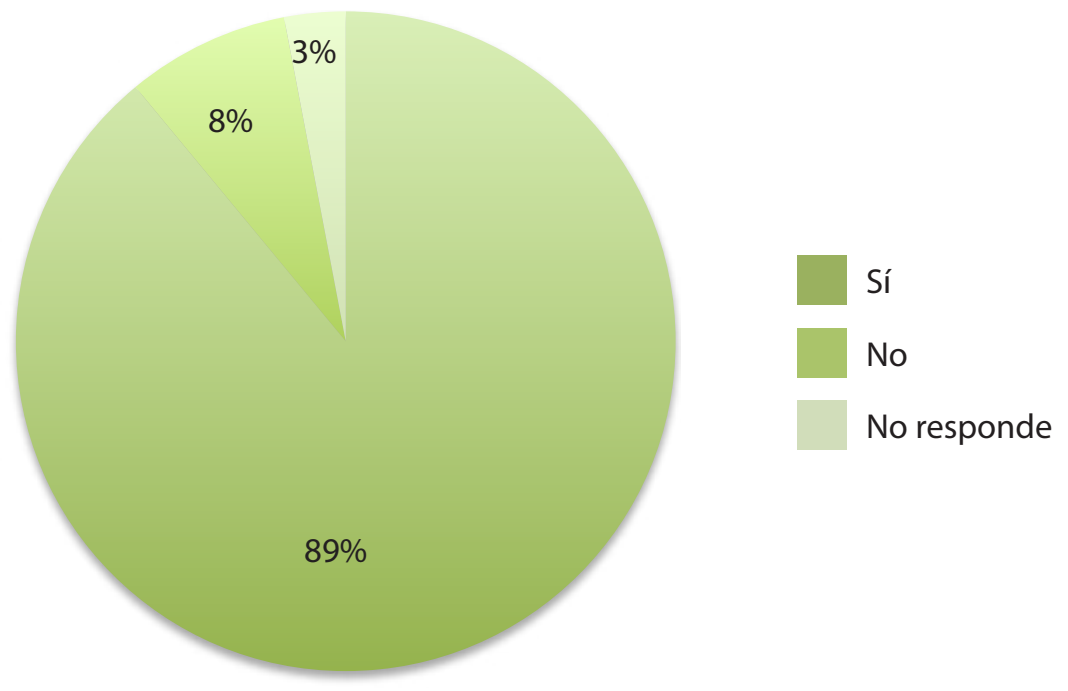

Figura 3: Experiencias que brinda la carrera con la niñez menor de 4 años. Elaboración propia.

Al preguntar dónde se obtuvieron estas experiencias de trabajo con la niñez menor de 4 años (Figura 4), un 2\% respondió que en la intervención pedagógica, otro 7\% en proyectos en el ambiente preescolar, desarrollados en la formación universitaria, por ejemplo, los cursos de intervención pedagógica en el aula preescolar y el curso de Proyecto Pedagógico en el Aula Preescolar (Plan de Estudios de la Carrera de Educación Preescolar, 2013), un 21\% en observaciones participantes en las visitas en el aula y la observación en diferentes contextos pedagógicos, un $29 \%$ considera que las obtiene de otros espacios y para finalizar un $31 \%$ indica haberla obtenido de cursos optativos, lo que evidencia que la población consultada apunta que su formación con niñez de 0 a 4 años no fue dada en el marco del plan de estudios de la carrera y proviene de otras fuentes que ya se han mencionado anteriormente.

En este aspecto es importante aclarar que esas experiencias que indican las estudiantes, según su opinión, provienen de cursos optativos que forman parte de la oferta de formación 
que se ofrece en la DEB, tal es el caso del curso optativo Un acercamiento al ciclo materno infantil: Retos y rol o el curso estimulación temprana y educación, como se observa más adelante en los resultados de las sugerencias de mejora propuestas por el estudiantado en la formación docente. Un dato importante en la reflexión consiste en que no son cursos incluidos dentro del plan de estudios de la Carrera de Educación Preescolar. Este tipo de oferta académica es opcional y solamente se cursa si el estudiantado lo elige dentro de su programa de estudios (Plan de Estudios en Pedagogía con énfasis en Educación Preescolar, 2013).

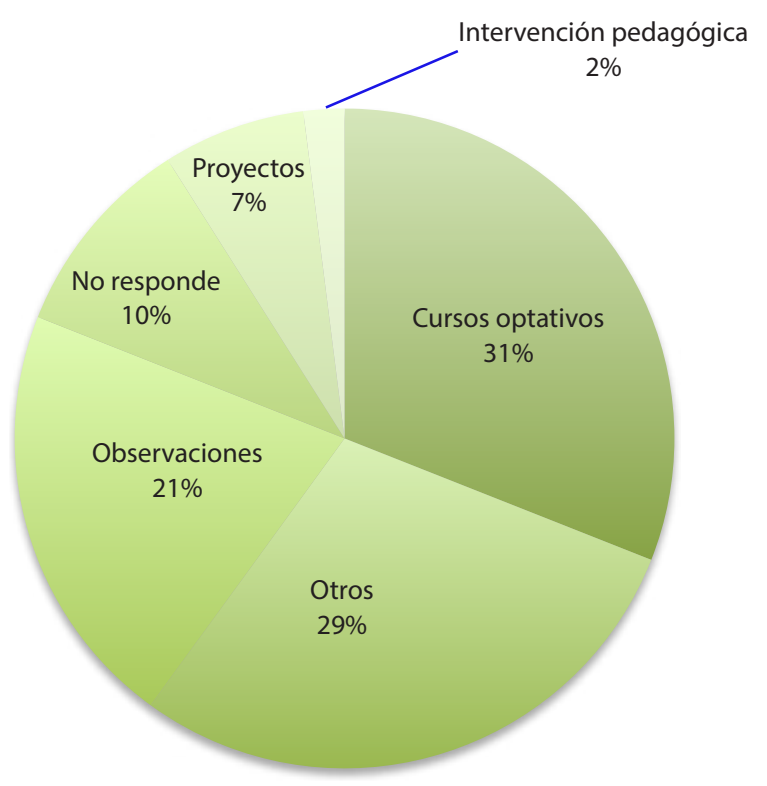

Figura 4: ¿Cómo se ha obtenido experiencia con la niñez menor de 4 años? Elaboración propia.

De igual forma, al consultar si en la carrera hay cursos para la atención y educación de menores de 4 años, un $33 \%$ respondió que sí, $46 \%$ que no y $21 \%$ que había muy pocos, como se muestra en la Figura 5. Los datos recopilados apuntan a que hay una percepción del estudiantado que afirma la necesidad de que dentro del Plan de Estudios de la Carrera de Educación Preescolar existan cursos específicos para la educación oportuna en los primeros tres años de vida. Este aspecto ya ha sido señalado por Ruiz, Castillo, Ramírez y Urdaneta (2015), cuando indican que la educación desde el nacimiento es un desafío nacional y que requiere de un tratamiento especial no solo desde los servicios que se ofrecen a los sectores usuarios y beneficiarios (familias, madres trabajadoras, niñez en condición de vulnerabilidad), sino también, desde la formación docente que se está ofreciendo en la oferta académica en las universidades. 
doi: http://dx.doi.org/10.15359/ree.21-1.9

URL: http://www.una.ac.cr/educare

CORREO: educare@una.cr

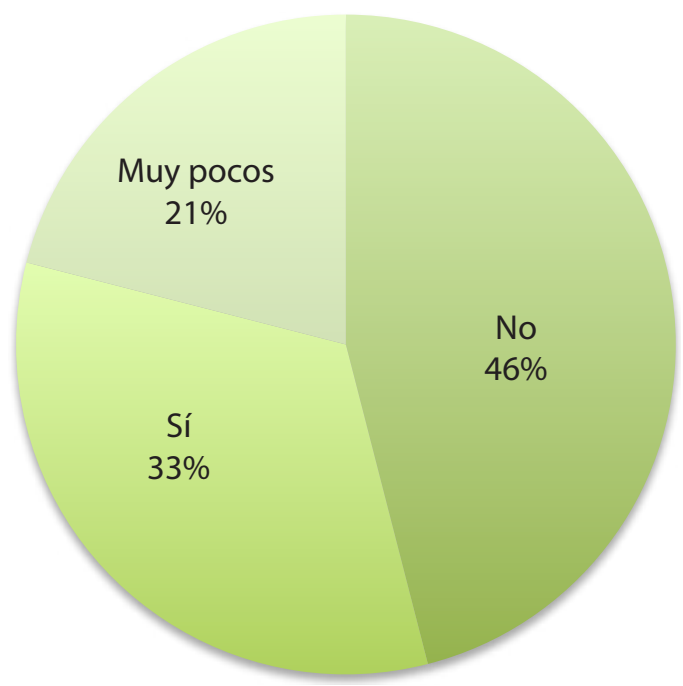

Figura 5: Cursos que aborden la temática de la niñez menor de 4 años. Elaboración propia.

La población investigada considera que tuvieron muy pocas oportunidades de formación para la niñez de 0 a 4 , se menciona como significativo que en el primer semestre de su formación en la licenciatura conocieron espacios formales y no formales, además, en algunos cursos en los cuales se les permitió trabajar en el sector privado y, sobre todo, en el curso optativo llamado Un acercamiento al ciclo materno infantil. Desde el proyecto de investigación Primera Infancia: Hacia una propuesta de formación contextualizada y pertinente (Ramírez et al., 2015), se encuentra que una de las prioridades manifiestas por parte del personal docente consultado en dicho estudio es la importancia de que, cuando se está formando al personal docente, se retomen la diversidad de espacios pedagógicos en los que se atiende a la niñez menor de 3 años. Una limitante señalada por Urdaneta (2013) es que la mayoría de intervenciones pedagógicas del estudiantado en formación se da en el ámbito preescolar público que atiende, en su mayoría, a niños y niñas mayores a los 4 años. Esto induce a suponer que las prácticas se desarrollan en este tipo de ambientes y que, por tanto, sea el referente empírico, es decir, su experiencia previa y cercana más implementada en la formación del personal docente de educación preescolar.

Esto es reiterado por la población consultada cuando expresa, entre sus necesidades, el que se pueda acceder a espacios diversos de atención y educación a la primera infancia (Tabla 1), que no sean solamente de la oferta que ofrece el Ministerio de Educación Pública del país.

Necesidades de formación expresadas por el estudiantado consultado. Como parte de la indagación se instó a las estudiantes a reflexionar sobre cuáles son las principales necesidades de formación que presentan para el trabajo con menores de cuatro años, sujetas a 
estas cuatro aristas: necesidades teóricas, necesidades curriculares, necesidades metodológicas y necesidades del trabajo con la familia y la comunidad. Para una mayor comprensión se dispuso la información obtenida en la siguiente matriz (Tabla 1).

Tabla 1: Necesidades de formación expresadas por el estudiantado

\begin{tabular}{|c|c|c|c|}
\hline Necesidades teóricas & $\begin{array}{l}\text { Necesidades } \\
\text { curriculares }\end{array}$ & $\begin{array}{l}\text { Necesidades } \\
\text { metodológicas }\end{array}$ & $\begin{array}{l}\text { Necesidades del trabajo con } \\
\text { la familia y la comunidad }\end{array}$ \\
\hline $\begin{array}{l}\text { - Mayor conocimiento } \\
\text { teórico acerca del } \\
\text { desarrollo del niño o } \\
\text { niña de } 0 \text { a } 4 \text { años. } \\
\text { - Agregar al plan de } \\
\text { estudios cursos para } \\
\text { profundizar temáticas } \\
\text { de psicología infantil, } \\
\text { nutrición, estimulación } \\
\text { temprana, neurociencia } \\
\text { y teorías emergentes en } \\
\text { la atención de población } \\
\text { de } 0 \text { a } 4 \text { años. } \\
\text { - Una base más sólida en } \\
\text { investigación. } \\
\text { - Conocimientos } \\
\text { sobre evaluación del } \\
\text { desarrollo en la niñez }\end{array}$ & $\begin{array}{l}\text { - Cursos para la carrera que } \\
\text { se centren propiamente } \\
\text { en esta área y abarquen, } \\
\text { por sobre todo, formas de } \\
\text { planificación curricular, } \\
\text { actividades y estrategias. } \\
\text { - Currículo no solamente } \\
\text { basado en el del MEP, } \\
\text { que dé paso a todo } \\
\text { el panorama que da } \\
\text { atención a la primera } \\
\text { infancia. } \\
\text { - Funcionamientos en } \\
\text { los centros (públicos o } \\
\text { privados) que atienden } \\
\text { a esta población. } \\
\text { - Fortalecimiento de } \\
\text { la atención de las } \\
\text { necesidades educativas. } \\
\text { - Inclusión del } \\
\text { idioma inglés como } \\
\text { competencia y } \\
\text { herramienta de trabajo. } \\
\text { - Realizar una revisión } \\
\text { para determinar los } \\
\text { cursos optativos, ya que } \\
\text { varios deberían estar } \\
\text { dentro de la carrera. } \\
\text { - Mayor coherencia en el } \\
\text { orden de los cursos de } \\
\text { carrera. }\end{array}$ & $\begin{array}{l}\text { - Mayor apertura para } \\
\text { asistir, realizar trabajos } \\
\text { y la práctica profesional } \\
\text { en espacios de atención } \\
\text { a la primera infancia. } \\
\text { - Formación en } \\
\text { estrategias didácticas } \\
\text { para la atención de esta } \\
\text { población. } \\
\text { - Conocer las } \\
\text { adaptaciones necesarias } \\
\text { para trabajar con la } \\
\text { población (temáticas } \\
\text { a abordar, tiempo de } \\
\text { atención, etc.). } \\
\text { - Identificar cuál de las } \\
\text { metodologías conocidas } \\
\text { es la indicada para esta } \\
\text { población. } \\
\text { - Conocer más acerca } \\
\text { del trabajo con niños y } \\
\text { niñas con necesidades } \\
\text { educativas espaciales } \\
\text { inmersos en el sistema } \\
\text { regular. }\end{array}$ & $\begin{array}{l}\text { - Un mayor enfoque de } \\
\text { trabajo conjunto con la } \\
\text { familia desde el inicio de } \\
\text { la carrera. } \\
\text { - Más proyectos, } \\
\text { espacios de acción u } \\
\text { otros que permitan } \\
\text { acercarse e involucrarse } \\
\text { mayormente con las } \\
\text { familias y la comunidad. } \\
\text { - Técnicas para la } \\
\text { comunicación asertiva } \\
\text { con padres y madres de } \\
\text { familia. }\end{array}$ \\
\hline
\end{tabular}

Nota: Elaboración propia. 
doi: http://dx.doi.org/10.15359/ree.21-1.9

URL: http://www.una.ac.cr/educare

CORREO: educare@una.cr

En relación con las necesidades teóricas se reitera la importancia de ahondar en el área de desarrollo humano en las edades comprendidas desde el nacimiento hasta los 4 años. Según la población consultada, es esencial que se contemplen, dentro del plan, temas como psicología infantil, nutrición, estimulación temprana, neurociencia y teorías emergentes en la atención de población de 0 a 4 años.

Esto plantea un desafío para el cuerpo docente encargado de impartir los cursos de la carrera, porque supone, también, un proceso de auto y mutua formación en estos temas que apuntan las estudiantes hacia una actualización de la oferta curricular que se les ofrece actualmente. Esto implica que se valore, entre otros aspectos, que durante los años 2013-2015, un porcentaje considerable (79\%) indicó que su experiencia laboral es con niñez menor a los 4 años (Figura 1, Figura 2).

Se señalan, por parte de las consultadas, otros puntos para el análisis, como el que se tome en cuenta que la formación no debe centrarse solo para la oferta de programas del Ministerio de Educación Pública, se hace un llamado a la diversificación existente en los servicios y programas que se ofrecen para la primera infancia en el país, así como la creciente demanda de una persona profesional en educación preescolar bilingüe.

Un aspecto de fondo que contempla el estudiantado es que se requiere de un estudio a profundidad del plan de estudios en relación con el orden de los cursos. Se considera que esta afirmación se hace, porque algunos que desarrollan la temática de desarrollo infantil están ubicados en el bachillerato y antes, en el diplomado, se han dado los cursos operativos de estrategias pedagógicas y ha pasado el período de la práctica profesional; debilidad ya señalada en la sistematización realizada por Urdaneta (2013).

Las necesidades metodológicas son otros de los puntos importantes a considerar dentro de la formación docente. El estudiantado plantea que es oportuno retomar la práctica profesional en espacios donde se atienda a la niñez desde el nacimiento, aunado al acompañamiento docente en su atención pedagógica. Se evidencia la falta de conocimiento en relación con el trabajo en espacios educativos que atienden bebés, en relación con los contenidos curriculares, el manejo de la dimensión temporal, la ambientación física y pedagógica y las interacciones docentes-estudiantes. La población consultada recomienda que desde el aula universitaria se oriente hacia metodologías pedagógicas más oportunas para la primera infancia.

Un tema recurrente también lo constituye la inclusión de los niños y niñas con alguna condición de discapacidad. Las estudiantes señalan que es necesario que este aspecto se incorpore con más fuerza.

Todo lo anterior permite visualizar a un estudiantado que se enfrenta a una realidad laboral compleja, para la cual hay demandas de un sujeto profesional docente en un encuadre 
de formación amplio y actualizado, preparado para las demandas de un contexto que requiere que la primera infancia sea atendida y educada desde otra posición epistemológica, que supere los enfoques asistenciales y técnicos.

Un aspecto que también fue señalado en la sistematización realizada por Urdaneta (2013) fue la incorporación del trabajo con la familia y la comunidad en el plan de estudios. La necesidad de un análisis contextualizado de la niñez en situación e interacción social y familiar enriquece y hace pertinente y situada la labor pedagógica. El estudiantado sugiere que se incorporen técnicas para lograr la participación familiar y comunal en el quehacer con los niños y niñas en los diferentes espacios pedagógicos.

Aspectos de mejora señalados por el estudiantado. La información recopilada también señala algunos de los aspectos que el estudiantado participante en el estudio considera que deben ser mejorados en la propuesta de formación profesional brindada por la carrera de Pedagogía con énfasis en Educación Preescolar.

- Mejor formación a nivel investigativo, de una manera real y funcional, desde el principio: "que se nos enseñe que es un marco teórico, por ejemplo y el uso de APA, es necesario un curso prácticamente de eso".

- Ofrecer la posibilidad de que las prácticas se realicen en centros privados, pues podrían traducirse en oportunidades laborales, esto desde la percepción del estudiantado, igualmente, es necesario salir del ambiente común e intentar experiencias en espacios distintos como la red de cuidado (REDCUDI), espacios emergentes, entre otros.

- El tema de las necesidades educativas debe ser abordado ampliamente. "Conocer lo básico de LESCO por ejemplo podría ayudar mucho".

- Es importante que se enseñe cómo responder a emergencias, tales como terremotos, amenazas de bomba, incendio, entre otros.

- Conocer todos los espacios y modalidades que atienden a la población menor de 4 años.

- Reestructuración de la malla curricular. "Buscar formas en que los contenidos de los cursos no sean tan repetitivos".

- Fortalecer el involucramiento del estudiantado con la familia y el contexto de los niños y niñas desde los primeros años de universidad.

- Iniciar proyectos de proyección comunal y social, donde se promueva la revalorización de la profesión de preescolar y la participación en programas de formación a padres y madres de familia.

- Realizar alianzas con instituciones gubernamentales y no gubernamentales de espacios educativos no formales que atienden a niños y niñas en riesgo social. 
doi: http://dx.doi.org/10.15359/ree.21-1.9

URL: http://www.una.ac.cr/educare

CORREO: educare@una.cr

- Promover el trabajo interdisciplinario, mediante la creación de grupos de trabajo; donde se realicen propuestas de mayor impacto en la vida de los niños y niñas, así como a nivel social.

- Dar un mayor énfasis al desarrollo cognitivo y socioemocional.

- Se recomienda la unificación de trabajos de los cursos de cada nivel.

- Algunos cursos optativos deben ser parte de la malla curricular de la carrera, "por ejemplo: Estimulación temprana, Un acercamiento al ciclo materno infantil"

- Focalizar el aprendizaje que se quiere de una profesional en educación preescolar frente a todas las demandas del mercado.

- Que los cursos de atención a las necesidades educativas especiales, propios de la carrera, sean reestructurados en función de la primera infancia, ya que se reciben cursos exactamente iguales entre las carreras.

- Ofrecer a las estudiantes una formación en el área de lecto escritura, ya que este se ha convertido en un contenido a desarrollar especialmente en los centros educativos privados.

- Incorporar psicología infantil y estimulación temprana con profesionales especialistas en la materia.

\section{Reflexiones finales}

Los principales hallazgos de los datos obtenidos indican que:

\section{En relación con la experiencia laboral de las estudiantes}

- A pesar de que la mayoría de las estudiantes participantes de este estudio ha trabajado con población menor de 4 años, consideran que la propuesta de formación ofrecida en su carrera no ofrece un acercamiento real con la niñez menor de 4 años y que muchas de las experiencias vividas con esta población se las han ofrecido otros espacios y cursos que no están en la malla curricular del plan (cursos optativos).

- Sauma (2012) lo indica: es necesario que toda propuesta de formación profesional apunte al conocimiento de la población de 0 a 4 años. En la realidad estudiada, el Plan de estudios de la carrera de Educación Preescolar debe responder a esta demanda, que no solo tiene incidencia en la formación universitaria, sino que es un requerimiento de las demandas sociopolíticas y de las tendencias del avance científico en el estudio del desarrollo humano. 


\section{En relación con las necesidades de formación encontradas}

- Las principales necesidades de formación señaladas por el estudiantado participante son las relacionadas con conocer más acerca del desarrollo infantil de la niñez menor de 4 años. También, se apunta que es muy importante conocer acerca de temáticas que contribuyan a potenciarel desarrollo integral deesta población comoloesla neurociencia, las necesidades educativas especiales en la primera infancia, la estimulación temprana, la piscología infantil y el idioma inglés, entre otras. En consecuencia, debe reflexionarse sobre estas necesidades formativas, para dar coherencia a los fundamentos y principios pedagógicos que sustentan la formación de formadores y formadoras en las nuevas tendencias de atención, educación y cuido de la primera infancia.

\section{En relación con las necesidades de mejora en el plan de estudios}

- En la parte metodológica, se insiste en la urgencia de que en la formación se incluyan espacios de intervención diversos que abarquen no solamente el sistema formal, sino también, el no formal, dado que estos son estos espacios en los que el estudiantado considera tiene mayores posibilidades laborales.

- Con respeto a lo curricular, el estudiantado considera que es necesario realizar una revisión de la malla curricular que permita un ordenamiento más adecuado para la formación y la inclusión de cursos específicos para tratar la niñez menor de 4 años, así como también la incorporación, a la malla curricular, de los cursos optativos, que se ofrecen en la División de Educación Básica y abordan esta temática.

- Un aspecto relevante con respecto a los datos recopilados en la revisión bibliográfica es que muchos de los aspectos señalados anteriormente coinciden con los obtenidos en una investigación realizada en la Universidad de Costa Rica, por Álvarez et al. (2011), que indican:

Con respecto a los planes de estudio de formación profesional, las entrevistadas muestran que, si bien estos han mejorado con el pasar del tiempo, aún deben ser fortalecidos para incorporar debilidades que tienen en cuanto a intervención oportuna, necesidades educativas especiales, elaboración de material didáctico, ética profesional y dominio del idioma inglés. Además, se concluye que la realización de prácticas y observaciones durante la formación resulta trascendental para el futuro desempeño profesional. (p. 17)

Es importante señalar que la exploración realizada ha permitido cumplir con el objetivo inicial de evidenciar algunos de los hallazgos más significativos en cuanto a necesidades de formación, se aportan elementos claves para revisar la propuesta ofrecida en la Universidad Nacional para la formación profesional en Pedagogía con énfasis en Educación Preescolar. 
doi: http://dx.doi.org/10.15359/ree.21-1.9

URL: http://www.una.ac.cr/educare

CORREO: educare@una.cr

Al observar estos elementos, resulta indispensable asumir una posición reflexiva y situada en la necesidad de una propuesta de formación contextualizada y pertinente, que permita, al estudiantado, enfrentar una realidad que ya no se puede evadir: La niñez necesita de atención y educación oportuna desde el nacimiento. Se requieren profesionales con preparación para el desarrollo de programas que superen las visiones asistencialistas y tengan claridad de la intencionalidad, el significado y la trascendencia de la atención y educación de la primera infancia.

\section{Referencias}

Álvarez, M., Arley, A., Gazel, A., González, M., Gutiérrez, K. y Vargas, A. (2011). Percepciones del personal docente en educación inicial sobre su profesión en el papel que desempeñan en la sociedad. Actualidades investigativas en la educación, 11(3), 1-23. doi: https://doi. org/10.15517/aie.v11i3.10216

Amador, L. H. (2007). Formación en tiempos presentes hacia pedagogías emergentes. Revista Latinoamericana de estudios educativos, 3(1), 41-63. Recuperado de http://www.redalyc. org/articulo.oa?id=134112603004

Chaves, L., Díaz, M., García, J., Rojas, G., Solís, N. (2007). Investigación-acción colaborativa: Un encuentro con el quehacer cotidiano del centro educativo para su transformación. San José, Costa Rica: INIE.

Elvir, A.P.y Asensio, C. (2006).Laatención yeducación de la primera infancia en Centroamérica:Desafíos y perspectivas. Recuperado de http://unesdoc.unesco.org/images/0014/001474/147488s.pdf

Eming, M., y Fujimoto, G. (2004). Desarrollo infantil temprano: Lecciones de los programas no formales. Acción Pedagógica, 13(2), 186-198. Recuperado de http://www.saber.ula.ve/ bitstream/123456789/17211/2/articulo7.pdf

Myers, R. G. (1999). Atención y desarrollo de la primera infancia en Latinoamérica y el Caribe: Una revisión de los diez últimos años y una mirada hacia el futuro. Revista lberoamericana de Educación, 22, 17-39. Recuperado de http://www.rieoei.org/rie22a01.PDF

Peralta, M. V. (2005). Nacidos para ser y aprender. Buenos Aires: Ediciones Infantojuvenil.

Programa Estado de la Nación. (2013). Cuarto informe estado de la educación (4a ed.). San José: Costa Rica. Autor. Recuperado de http://www.estadonacion.or.cr/files/biblioteca virtual/ educacion/004/6-Cap-2.pdf

Ramírez, P., Ruiz, S. y Castillo, R. (2015). Primera infancia: Hacia una propuesta de formación profesional contextualizada y pertinente (proyecto). Heredia: Costa Rica: Universidad Nacional, CIDE. 
Ruiz, S., Castillo, R., Ramírez, P., Urdaneta, M. (2015). La atención y educación de la primera infancia: Un derecho pendiente en la sociedad costarricense para la población de 0-3 años. Revista Latinoamericana de Derechos Humanos, 26(1), 166-177. doi: https://doi. org/10.15359/rldh.26-1.8

Sauma, P. (2012). Cuarto informe del estado de la educación. Informe final. Perfil de la población de 0 a 6 años y escenarios para su atención dentro y fuera del sistema educativo. San José: Costa Rica: Programa Estado de la Nación. Recuperado de http://estadonacion.or.cr/files/ biblioteca virtual/educacion/004/Sauma poblacion-de-0-a-6-anos.pdf

Urdaneta, M. (2013). Informe de la Comisión de Diseño Curricular. Heredia, Costa Rica: Universidad Nacional, CIDE, División de Educación Básica. Manuscrito inédito.

Villegas-Reimers, E. (2002). Formación docente en los Estados Unidos de Norteamérica:Tendencias recientes en sus prácticas y políticas. En Unesco (Ed.), Formación docente: Un aporte a la discusión. La experiencia de algunos países (pp. 59-90). Santiago, Chile: Unesco/Orealc.

Zapata, B. y Ceballos, L. (2010). Opinión sobre el rol y perfil del educador para la primera infancia. Revista Latinoamericana de Ciencias Sociales, Niñez y Juventud, 8(2), 1069-1082. Recuperado de http://www.redalyc.org/pdf/773/77315155021.pdf 